\title{
GENERAL RELATIONS BETWEEN BERNOULLI, EULER, AND ALLIED POLYNOMIALS*
}

BY

E. T. BELL

1. Introduction. The derivation of a complete set of general relations $(\$ \$ 5-9,10)$ between the polynomials of Bernoulli, Euler, Genocchi, and Lucas, is reduced by the symbolic method $(\$ \S 3,4)$ to elementary algebraical operations (addition, multiplication, resolution into partial fractions) on four rational functions of the form $N(t) / D(t)$, where $N, D$ are polynomials of degree $\leqq 2$ in $t$. In $\S 9$ it is shown that the relations imply a complete set of relations between the Bernoulli and allied numbers. The second of the transformations in $\$ 10$, by specifying $h$, gives relations between the polynomials (or associated numbers) when their ranks are in arithmetical progression with any positive common difference.

2. Notation. The even-suffix notation is used for the numbers $B, E, G, R$ of Bernoulli, Euler, Genocchi, and Lucas:

$$
\begin{aligned}
& B_{0}=1, \quad B_{1}=-\frac{1}{2}, B_{2 s+1}=0(s>0), B_{2}=\frac{1}{6}, \quad B_{4}=-\frac{1}{30}, B_{6}=\frac{1}{42}, \cdots ; \\
& E_{0}:=1, \quad E_{2 s+1}=0(s \geqq 0), \quad E_{2}=-1, \quad E_{4}=5, \quad E_{6}=-61, \cdots ; \\
& G_{0}:=0, \quad G_{1}=1, \quad G_{2 s+1}=0(s>0), \quad G_{2}=-1, \quad G_{4}=1, \quad G_{6}=-3 ; \\
& R_{0}=\frac{1}{2}, \quad R_{2 s+1}=0(s \geqq 0), \quad R_{2}=-\frac{1}{6}, \quad R_{4}=\frac{7}{30}, \quad R_{6}=-\frac{31}{42} ; \\
& G_{s}=2\left(1-2^{s}\right) B_{s}, \quad R_{s}=\left(1-2^{s-1}\right) B_{8}(s \geqq 0) ;
\end{aligned}
$$

the $E, G$ are integers. Let $x$ denote a complex variable. The above numbers have the following symbolic generators, in which the expansions of the exponentials converge absolutely for some $|x| \neq 0$ :

$$
\frac{x}{e^{x}-1}=e^{B x}, \quad \frac{2 e^{x}}{e^{2 x}+1}=e^{E x}, \quad \frac{2 x}{e^{x}+1}=e^{G x}, \quad \frac{x e^{x}}{e^{2 x}-1}=e^{R x} .
$$

Let $u$ be a complex variable and $\alpha$ the umbra of the sequence $\alpha_{n}(n=0,1$, $2, \cdots)$. The Appell polynomial of degree $n$ in $u$ with the base $\alpha$ is

$$
(u+\alpha)^{n} \equiv \sum_{s=0}^{n}{ }_{n} C_{s} \alpha_{s} u^{n-s} \quad\left({ }_{0} C_{0}=1\right) ;
$$

\footnotetext{
* Presented to the Society, September 13, 1935; received by the editors December 4, 1934.
} 
its generator is obtained by multiplying the generator $e^{x \alpha}$ of $\alpha$ by $e^{x u}$, thus, $e^{x u} e^{x \alpha}=e^{x(u+\alpha)}$.

The Appell polynomials in $u$ with the respective bases $B, E, G, R$ are (here) called the Bernoulli, Euler, Genocchi, and Lucas polynomials in $u$ :

$\beta_{n}(u) \equiv(u+B)^{n}, \quad \eta_{n}(u) \equiv(u+E)^{n}, \quad \gamma_{n}(u) \equiv(u+G)^{n}, \quad \rho_{n}(u) \equiv(u+R)^{n}$.

These definitions of the polynomials, instead of any of the numerous slightly different definitions in the literature, are chosen on account of the symmetry and simplicity of all calculations with the polynomials consequent upon their use. Other definitions can be readily translated into terms of these if necessary.

From the generators of $B, E, G, R$ we write down those of $\beta(u), \eta(u), \gamma(u)$, $\rho(u)$ :

$$
\begin{gathered}
t \equiv e^{x}, \quad w \equiv e^{x u} ; \\
\frac{x w}{t-1}=e^{x \beta(u)} \equiv P(t) \equiv P, \\
\frac{2 w t}{t^{2}+1}=e^{x \eta(u)} \equiv Q(t) \equiv Q, \\
\frac{2 x w}{t+1}=e^{x \gamma(u)} \equiv S(t) \equiv S, \\
\frac{x w t}{t^{2}-1}=e^{x \rho(u)} \equiv T(t) \equiv T .
\end{gathered}
$$

The generators are absolutely convergent for $|x|,|u|$ properly restricted and $\neq 0$.

Let $f(h)$ denote either a polynomial in the complex variable $h$ or, if absolutely convergent for some $h \neq 0$, a power series in $h$. The results of substituting $z$ for $h$ in the successive derivatives $f^{\prime}(h), f^{\prime \prime}(h), \ldots$ of $f(h)$ with respect to $h$ will be written $f^{\prime}(z), f^{\prime \prime}(z), \cdots$. This applies in particular if $z$ contains umbrae.

All of the foregoing notation will be used without further reference.

3. Order of relations; reductions. The umbrae $\alpha, \sigma$ are said to be distinct if and only if $\alpha_{n} \neq \sigma_{n}$ for some integer $n \geqq 0$. Let $h, a, \cdots, s$ be ordinaries (complex numbers) and $\alpha, \cdots, \sigma$ umbrae. Symbolically, $(h \alpha)^{n}$ means $h^{n} \alpha_{n}$. A linear expression of the form $h+a \alpha+\cdots+s \sigma$ is said to be of order $p$ (in the umbrae) if precisely $p$ umbrae $\alpha, \cdots, \sigma$ occur in it. The order of a relation involving functions of the form $f(h+a \alpha+\cdots+s \sigma)$ is by definition the highest order of any expression occurring as an argument of $f$ in the relation. 
To find relations of order $p$ in $\beta(u), \eta(u), \gamma(u), \rho(u)$ we multiply together precisely $p$ of their generators (if $p>4$, at least one generator will occur to a power $>1$ ). The product of generators, considered as a function of $t$, is then separated into partial fractions, from which it is easy to exhibit the product as a linear homogeneous function of the generators in the product, with coefficients of the form $H(x, w, t)$, where $H$ is a rational function. From the result we can write down a relation involving a finite number of functions $f$ and their derivatives $f^{\prime}, f^{\prime \prime}, \ldots$ in which the argument of one function is of order $p$ and the arguments of the rest are of order 1. One example of the simple process of reduction by which the results stated later were obtained will suffice.

To reduce $P Q$ to a linear homogeneous function of $P, Q$ we have

$$
\begin{aligned}
P Q & =\frac{2 x w^{2} t}{(t-1)\left(t^{2}+1\right)} ; \\
\frac{t}{(t-1)\left(t^{2}+1\right)} & =\frac{1}{2(t-1)}-\frac{t-1}{2\left(t^{2}+1\right)} ; \\
P Q & =w \frac{x w}{t-1}-\frac{(t-1) x w}{2 t} \frac{2 w t}{t^{2}+1}, \\
& =w P-\frac{(t-1) x w}{2 t} Q ; \\
2 t P Q & =2 w t P-x w(t-1) Q .
\end{aligned}
$$

4. Derivation of general relations. Let $h, x, a, d, \cdots, s, a^{\prime}, d^{\prime}, \cdots, s^{\prime}$ be ordinaries, $\alpha, \delta, \cdots, \sigma$ umbrae $\left(\alpha^{n}=\alpha_{n}\right.$ for $n=0,1, \cdots, \alpha^{m}=\alpha_{m}=0$ for $m<0$, and similarly for $\delta, \cdots, \sigma)$, and $i, j$, integers $\geqq 0$. Let

$$
a^{\prime} e^{x \alpha}=d^{\prime} x^{i} e^{x \delta}+\cdots+s^{\prime} x^{i} e^{x \sigma} .
$$

be an identity in $x$. Multiply throughout by $e^{x h}$, and equate coefficients of $x^{n}$. Then, with $D_{h^{p}} \equiv d^{p} / d h^{p}$, we have, for $n=0,1,2, \cdots$,

$$
a^{\prime}(h+\alpha)^{n}=D_{h}^{i} d^{\prime}(h+\delta)^{n}+\cdots+D_{h}{ }^{i} s^{\prime}(h+\sigma)^{n} ;
$$

and hence, if $f(x)=\sum_{n=0} c_{n} x^{n}$,

$$
a^{\prime} f(h+\alpha)=d^{\prime} f^{(i)}(h+\delta)+\cdots+s^{\prime} f^{(j)}(h+\sigma),
$$

where $f^{(p)}$ denotes the $p$ th derivative (as in $\$ 2$ ). Obviously the final relation can be written down at once from the given identity.

For example, the identity at the end of $\S 3$ is

$$
2 e^{x} e^{x(\beta(u)+\eta(u))}=2 e^{x u} e^{x} e^{\beta(u)}-x e^{x u}\left(e^{x}-1\right) e^{\eta(u)} ;
$$


that is,

$$
2 e^{x(1+\beta(u)+\eta(u))}=2 e^{x(u+1+\beta(u))}-x\left[e^{x(u+1+\eta(u))}-e^{x(u+\eta(u))}\right]
$$

hence

$$
\begin{aligned}
2 f(h+\beta(u)+\eta(u)+1)= & 2 f(h+\beta(u)+u+1) \\
& -f^{\prime}(h+\eta(u)+u+1)+f^{\prime}(h+\eta(u)+u) .
\end{aligned}
$$

We consider first relations in which no umbra occurs twice in the argument of any function in the relation.

5. Relations of order 1 . From the generators of $\beta, \eta, \gamma, \rho$ in $\$ 2$ we have

$$
\begin{aligned}
(t-1) P & =x w, & & \left(t^{2}+1\right) Q=2 w t, \\
(t+1) S & =2 x w, & \left(t^{2}-1\right) T & =x w t .
\end{aligned}
$$

Hence (as in \$3) we write down the general relations

$$
\begin{aligned}
& f(h+\beta(u)+1)-f(h+\beta(u))=f^{\prime}(h+u) ; \\
& f(h+\eta(u)+2)+f(h+\eta(u))=2 f(h+u+1) ; \\
& f(h+\gamma(u)+1)+f(h+\gamma(u))=2 f^{\prime}(h+u) ; \\
& f(h+\rho(u)+2)-f(h+\rho(u))=f^{\prime}(h+u+1) .
\end{aligned}
$$

Again, directly from the generators,

$$
4 T(x)=2 P(x)+S(x), \quad 2 w T(x)=t P(2 x), \quad 2 x w Q(x)=t S(2 x) ;
$$

(5) $4 f(h+\rho(u))=2 f(h+\beta(u))+f(h+\gamma(u))$;

(6) $2 f(h+\rho(u)+u)=f(h+2 \beta(u)+1)$;

(7) $2 f^{\prime}(h+\eta(u)+u)=f(h+2 \gamma(u)+1)$.

The six possible pairs chosen from $\beta, \eta, \gamma, \rho$ give relations of the first order from

$$
P(x)=\frac{x\left(t^{2}+1\right)}{2 t(t-1)} Q(x)=\frac{t+1}{2(t-1)} S(x)=\frac{t+1}{t} T(x)
$$

(8) $2 f(h+\beta(u)+2)-2 f(h+\beta(u)+1)=f^{\prime}(h+\eta(u)+2)+f^{\prime}(h+\eta(u))$;

(9) $2 f(h+\beta(u)+1)-2 f(h+\beta(u))=f(h+\gamma(u)+1)+f(h+\gamma(u))$;

(10) $f(h+\beta(u)+1)=f(h+\rho(u)+1)+f(h+\rho(u))$;

(11) $f^{\prime}(h+\eta(u)+2)+f^{\prime}(h+\eta(u))=f(h+\gamma(u)+2)+f(h+\gamma(u)+1)$;

(12) $f^{\prime}(h+\eta(u)+2)+f^{\prime}(h+\eta(u))=2 f(h+\rho(u)+2)-2(f+\rho(u))$;

(13) $f(h+\gamma(u)+1)=2 f(h+\rho(u)+1)-2 f(h+\rho(u))$.

These also follow easily from (1)-(4). 
6. Relations of order 2. These are given by the reductions of $P Q, P S$, $P T, Q S, Q T, S T$. We have

$$
\begin{aligned}
2 t P Q & =2 w t P-x w(t-1) Q ; \\
2 P S & =2 x w P-x w S, \\
t P S & =2 x w T ; \\
2 t(t-1) P T & =x w t P+x w(t-1) T ; \\
t Q S & =x w(t+1) Q-w t S ; \\
2 t Q T & =x w Q+2 w T, \\
Q(x) T(x) & =T(2 x) ; \\
2 t(t+1) S T & =x w t S+2 x w(t+1) T .
\end{aligned}
$$

To these correspond respectively

(14) $2 f(h+\beta(u)+\eta(u)+1)=f(h+u+\beta(u)+1)$

$$
-f^{\prime}(h+u+\eta(u)+1)+f^{\prime}(h+u+\eta(u)) \text {; }
$$

(15) $2 f(h+\beta(u)+\gamma(u))=2 f^{\prime}(h+u+\beta(u))-f^{\prime}(h+u+\gamma(u))$,

(16) $f(h+\beta(u)+\gamma(u)+1)=2 f^{\prime}(u+\rho(u))$;

(17) $2 f(h+\beta(u)+\rho(u)+2)-2 f(h+\beta(u)+\rho(u)+1)$

$$
=f^{\prime}(h+\beta(u)+u+1)+f^{\prime}(h+\rho(u)+u+1)-f^{\prime}(h+u+\rho(u)) ;
$$

(18) $f(h+\eta(u)+\gamma(u)+1)=f^{\prime}(h+\eta(u)+u+1)$

$$
+f^{\prime}(h+\eta(u)+u)-f(h+\gamma(u)+u+1) \text {; }
$$

(19) $2 f(h+\eta(u)+\rho(u)+1)=f^{\prime}(h+\eta(u)+u)+2 f(h+\rho(u)+u)$,

(20) $f(h+\eta(u)+\rho(u))=f(h+2 \rho(u))$;

(21) $2 f(h+\gamma(u)+\rho(u)+2)+2 f(h+\gamma(u)+\rho(u)+1)$

$$
\begin{aligned}
= & f^{\prime}(h+\gamma(u)+u+1)+2 f^{\prime}(h+\rho(u)+u+1) \\
& +2 f^{\prime}(h+\rho(u)+u) .
\end{aligned}
$$

7. Relations of orders 3, 4. The reductions of $Q S T, P S T, P Q T, P Q S$ provide several alternatives; we choose the simplest.

$$
\begin{aligned}
& 2 t(t+1) Q S T=-x^{2} w^{2}(t+1)^{2} Q+x w^{2} t S+2 x w^{2} t(t+1) T \\
& \left(t^{2}-1\right) P S T=2 x^{2} w^{2} T ; \\
& 4 t(t-1) P Q T=2 x w^{2} t P-x^{2} w^{2}\left(t^{2}-1\right) Q+2 x w^{2} t(t-1) T \\
& 2 P Q S=2 x w^{2} P-2 x^{2} w^{2} Q+x w^{2} S .
\end{aligned}
$$

The simplest relation of order 4 is 


$$
\begin{aligned}
& 2 t\left(t^{2}-1\right) P Q S T=2 x^{2} w^{3}\left(t^{2}+1\right) T-x^{3} w^{3} t\left(t^{2}-1\right) Q . \\
& 2 f(h+\eta(u)+\gamma(u)+\rho(u)+2)+2 f(h+\eta(u)+\gamma(u)+\rho(u)+1) \\
& =-f^{\prime \prime}(h+\eta(u)+2 u+2)-2 f^{\prime \prime}(h+\eta(u)+2 u+1) \\
& -f^{\prime \prime}(h+\eta(u)+2 u)+f^{\prime}(h+\gamma(u)+2 u+1) \\
& +2 f^{\prime}(h+\rho(u)+2 u+2)+2 f^{\prime}(h+\rho(u)+2 u+1) \text {; } \\
& \text { (23) } f(h+\beta(u)+\gamma(u)+\rho(u)+2)-f(h+\beta(u)+\gamma(u)+\rho(u)) \\
& =2 f^{\prime \prime}(h+\rho(u)+2 u) \text {; } \\
& 4 f(h+\beta(u)+\eta(u)+\rho(u)+2)-4 f(h+\beta(u)+\eta(u)+\rho(u)+1) \\
& =2 f^{\prime}(h+\beta(u)+2 u+1)-f^{\prime \prime}(h+\eta(u)+2 u+2) \\
& +f^{\prime \prime}(h+\eta(u)+2 u)+2 f^{\prime}(h+\rho(u)+2 u+2) \\
& -2 f^{\prime}(h+\rho(u)+2 u+1) \text {; } \\
& \text { (25) } f(h+\beta(u)+\eta(u)+\gamma(u))=2 f^{\prime}(h+\beta(u)+2 u)-2 f^{\prime \prime}(h+\eta(u)+2 u) \\
& +f^{\prime}(h+\gamma(u)+2 u) \text {; } \\
& 2 f(h+\beta(u)+\eta(u)+\gamma(u)+\rho(u)+3) \\
& -2 f(h+\beta(u)+\eta(u)+\gamma(u)+\rho(u)+1) \\
& =2 f^{\prime \prime}(h+\rho(u)+3 u+2)+2 f^{\prime \prime}(h+\rho(u)+3 u) \\
& -f^{\prime \prime \prime}(h+\eta(u)+3 u+3)+f^{\prime \prime \prime}(h+\eta(u)+3 u+1) \text {. }
\end{aligned}
$$

8. Relations with repeated umbrae. In an expression involving $\alpha+\alpha$ $+\cdots+\alpha$, where the umbra $\alpha$ is repeated precisely $n$ times, the $\alpha$ 's are replaced by $\alpha^{\prime}, \alpha^{\prime \prime}, \ldots$ respectively until after all exponents have been lowered to suffixes, when all accents are dropped, thus $\alpha^{\prime s}=\alpha^{\prime \prime s}=\cdots=\alpha_{s}$; the expression $\alpha+\alpha+\cdots+\alpha\left(n \alpha^{\prime}\right.$ s) will be written $\alpha^{(n)}$. As before, $f^{(n)}$ denotes the $n$th derivative of $f$ (as in \$2).

We have $x^{n} w^{n}=(t-1)^{n} P^{n} ;$ hence

$$
f^{(n)}(h+n u)=\sum_{s=0}^{n}(-1){ }_{n} C_{s} f\left(h+\beta^{(n)}(u)+n-s\right) .
$$

Similarly

$$
\begin{aligned}
2^{n} f(h+n u+n) & =\sum_{s=0}^{n}{ }_{n} C_{s} f\left(h+\eta^{(n)}(u)+2 n-2 s\right) \\
2^{n} f^{(n)}(h+n u) & =\sum_{s=0}^{n}{ }_{n} C_{s} f\left(h+\gamma^{(n)}(u)+n-s\right) ; \\
f^{(n)}(h+n u+n) & =\sum_{s=0}^{n}(-1)^{s} C_{s} f\left(h+\rho^{(n)}(u)+2 n-2 s\right) .
\end{aligned}
$$


For $n=1$, these become (1)-(4). The generators of $\beta^{(n)}(u), \eta^{(n)}(u), \gamma^{(n)}(u)$, $\rho^{(n)}(u)$ being $P^{n}, Q^{n}, S^{n}, T^{n}$ respectively, we could proceed as before to find relations of given orders for the $\beta^{(n)}(u), \cdots(n=1,2, \cdots)$.

9. Relations for $B, E, G, R$. From the generators in $\$ 2$ it is clear that $P, Q, S, T$ become the generators of $B, E, G, R$ respectively when $w=1$. Hence in (1)-(30) we may replace $u$ by 0 and $\beta(u), \eta(u), \gamma(u), \rho(u)$ by $B, E$, $G, R$ respectively.

10. Transformations. Considering $P \equiv P(x, u)$ as a function of two variables $x, u$, and similarly for $Q, S, T$, we easily find that all the rational transformations of $x, u$ which leave $P, Q, S, T$ invariant to within constant factors are the following:

$$
\begin{array}{ll}
P(x, u)=P(-x, 1-u), & Q(x, u)=Q(-x,-u), \\
S(x, u)=-S(-x, 1-u), & T(x, u)=T(-x,-u) .
\end{array}
$$

Hence

$$
\begin{array}{rlrl}
e^{x \beta(u)} & =e^{-x \beta(1-u)}, & & e^{x \eta(u)}=e^{-x \eta(-u)}, \\
e^{x \gamma(u)}=-e^{-x \gamma(1-u)}, & e^{x \rho(u)}=e^{-x \rho(-u)} .
\end{array}
$$

Multiplying each of these on both sides by $e^{x(h+r u+s)}$, where $h, r, s$ are ordinaries, and equating coefficients of $x^{n}(n=0,1, \ldots)$ in the results, we get

$$
\begin{aligned}
& (h+\beta(u)+r u+s)^{n}=(h-\beta(1-u)+r u+s)^{n}, \\
& (h+\eta(u)+r u+s)^{n}=(h-\eta(-u)+r u+s)^{n}, \\
& (h+\gamma(u)+r u+s)^{n}=-(h-\gamma(1-u)+r u+s)^{n}, \\
& (h+\rho(u)+r u+s)^{n}=(h-\rho(-u)+r u+s)^{n} ;
\end{aligned}
$$

whence, $f$ being as before, we have

$$
\begin{aligned}
& f(h+\beta(u)+r u+s)=f(h-\beta(1-u)+r u+s) ; \\
& f(h+\eta(u)+r u+s)=f(h+\eta(-u)+r u+s) ; \\
& f(h+\gamma(u)+r u+s)=-f(h-\gamma(1-u)+r u+s) ; \\
& f(h+\rho(u)+r u+s)=f(h-\rho(-u)+r u+s),
\end{aligned}
$$

and similarly for $f^{\prime}, f^{\prime \prime}, \ldots$. The arguments in (1)-(26) are of the form $h+\alpha(u)+r u+s(\alpha=\beta, \eta, \gamma, \rho)$, for $r, s$ properly chosen. Thus (31)-(34) enable us to write down from each of (1)-(26) at least one more general relation. The like, with obvious modifications, applies to (27)-(30).

Another type of transformation produces relations from which follow (as special cases) relations between functions $\beta_{n}(u), \ldots$ whose ranks $n$ are in arithmetical progression. Let $m$ be a positive integer, and let $f, f^{(m)}$ be as before. Write 


$$
F_{m, s}(k) \equiv \sum_{j=0} \frac{f^{(j m+s)}(k)}{(j m+s) !} \quad(s=0,1, \cdots, m-1),
$$

$F_{m, 8}^{\prime}(z) \equiv$ the result of substituting $z$ for $k$ in $D_{k} F_{m, s}(k)$, etc. Then, in any of the relations (1)-(26) we may replace $f(k)$ by $F_{m, s}(k), f^{\prime}(k)$ by $F_{m, s}^{\prime}(k), f^{\prime \prime}(k)$ by $F_{m, s}^{\prime \prime}(k)$, etc., where, as above, $k \equiv h+\alpha(u)+r u+s$, or the corresponding argument on the right of (31)-(34).

To see this, replace $h$ by $h+\mu$ in the argument of $f(h+\alpha(u)+r u+s)$, where $\mu$ is a primitive $m$ th root of unity, and expand (as a function of $h$ ) by MacLaurin's theorem,

$$
f(\mu+k)=\sum_{s=0}^{m-1} \mu^{s} F_{m, s}(k), \quad k \equiv h+\alpha(u)+r u+s .
$$

Proceed similarly with each $f, f^{\prime}, \cdots$ in a given relation. Since $1, \mu, \mu^{2}, \cdots$, $\mu^{m-1}$ are linearly independent, the result follows.

Added, March 27, 1935. In a recent abstract* D. H. Lehmer reports recurrences for Bernoulli numbers with gaps. These, apparently, are of a different type from those given by the above for special values of $m$.

* Bulletin of the American Mathematical Society, vol. 40 (1934), p. 51.

California Institute of Technology, Pasadena, Calitr. 